\title{
Editorial
}

\section{LA PROFESIONALIZACIÓN DE LA ACTIVIDAD DOCENTE EN LAS FACULTADES DE DERECHO COLOMBIANAS. VICISITUDES ACTUALES}

\author{
Juan Carlos Villalba Cuéllar (Editor) \\ Andrés González Serrano (Coeditor)
}

\begin{abstract}
El presente editorial reflexiona acerca de la enseñanza del derecho, oficio que sus autores escogieron con vocación decidida, pero que tiene unos contornos en el contexto colombiano que merecen unas palabras, siendo más específicos, se trata del ejercicio de la enseñanza del derecho como actividad profesionalizada, esto es, de tiempo completo. Huelga decir de antemano que por tratarse de reflexiones, lo que digan sus autores solamente compromete su opinión, la cual, como siempre, se considera abierta a discusión o sujeta a posturas contrarias.
\end{abstract}

El objetivo es poner de manifiesto algunas vicisitudes del oficio, que cualquiera que sea su origen, afectan de modo significativo la actividad docente universitaria cuando se realiza como una profesión. Para empezar debe decirse que todos aquellos que enseñaron derecho a las generaciones de abogados hasta tiempos recientes, no eran profesores de derecho en el sentido actual, eran abogados o jueces que dictaban clases. Eso no significa que lo hicieran mal, por el contrario, la mayoría de ellos lo hicieron y lo hacen aún muy bien; tampoco eso los descalificaba, pues muchos de ellos, a fuerza del tiempo, consolidaron esa vocación y nadie podría quitarles hoy el título de "profesores de derecho", ni más faltaba.

Aunque la enseñanza del derecho seguía siendo un apéndice de sus actividades profesionales, pues por más que quisieran, no era viable dedicarse de lleno a la enseñanza, ni el contexto estaba preparado para ello. Fueron "profesores de derecho" en una época en que esa labor no existía realmente en Colombia y procuraron hacerlo de la mejor manera. A ellos, de paso, nuestro sincero reconocimiento. La profesión de "docente investigador" con mayor razón era algo impensable. Lo que se evidencia entonces, es un proceso de evolución de la actividad docente que toda sociedad vive en algún momento y en las facultades de derecho del país se está experimentando en la actualidad.

Empero, los tiempos cambian y los escenarios también, y desde hace unos años por gracia de las políticas del Ministerio de Educación Nacional (MEN), en las facultades de derecho comenzaron a aparecer o a hacerse visibles personajes a los que se denominó "profesores de derecho", agregándoseles el apellido de "tiempo completo" o "dedicación completa". Varias prestigiosas universidades ya contaban con profesores de tal condición desde algún tiempo atrás, tomando la delantera, pero estos seguían siendo unos individuos extraños. Todavía ese "tiempo completo" ciertos profesores lo pronuncian entre labios, para que no se escuche alto a qué se dedican. Hoy, en las facultades de derecho colombianas conviven esas dos categorías de educadores, los profesores a la vieja usanza y los "profesores de tiempo completo". El profesor Molina (2014) en un artículo reciente se refiere a un choque generacional que suscita tensiones en las facultades de derecho.

Se evidencia así, que hoy por hoy en Colombia la puerta está abierta para que la actividad de "profesor de derecho" en el ámbito de las instituciones de educación superior, sea una verdadera opción profesional, lo cual se constituye ciertamente en un avance, al valorársele como el eje central de la universidad (López, 2008). No obstante, para que logremos tener verdaderos profesores de derecho las condiciones en su totalidad no están aún dadas, por tres factores a los que nos referiremos:(i) por la condición misma de quienes 
son profesores, (ii) por la consideración que las universidades tienen de sus docentes y (iii) por la situación del profesor investigador.

(i) La condición del profesor de derecho en Colombia.

- El rol de las diferentes clases de profesores en una facultad de derecho.

Decía un docente de una universidad pública en la década de los noventa, de esos que fueron precursores en ser educadores de dedicación total, que: "si los jueces dictan clases en sus ratos libres, pues los profesores deberíamos dictar sentencias en nuestros ratos libres". Y la frase pone en evidencia algo que sucede comúnmente, muchos de los "profesores de derecho" son jueces, funcionarios o litigantes de manera prevalente. La discusión acerca de proscribir su presencia en las facultades de derecho sería absurda, ellos son necesarios e importantes allí, su praxis profesional les permite "transmitir" a los estudiantes conocimientos y experiencias fruto del ejercicio profesional, cúmulo de muchos años, lo que de antemano es muy valioso para un aprendiz del derecho que aún no se enfrenta con la vida real. Esa discusión se descarta.

Lo que no podría seguir siendo admisible, y debe dejar de ocurrir en cumplimiento de las políticas del MEN, es que estos constituyan la totalidad del personal docente de una facultad de derecho. Eso puede tener muchas explicaciones, pero una de ellas es que para que en una universidad haya una verdadera "comunidad académica", es imprescindible que los profesores se dediquen a los estudiantes, y un docente que entra a una facultad tan solo a dictar la cátedra no tiene la posibilidad de hacerlo. Se requieren, por el contrario, profesionales que se consagren al estudio del derecho y sus problemas, así como a la práctica docente, desde todas sus perspectivas, desde la preparación de clases, el ejercicio tutorial con los estudiantes, la investigación, etc. Así mismo es imprescindible un grupo humano preparado para colaborar con la correcta administración de una facultad, en asuntos como la reflexión curricular, la optimización de microcurrículos, la mejora de praxis docentes, el análisis de estrategias para el posicionamiento de la facultad y de los educandos en el entorno social, el cumplimiento de estándares obligatorios y voluntarios de calidad académica (acreditación), la internacionalización, la formación continua, entre otros aspectos. Muchas de estas tareas también forman parte del quehacer docente, el cual no se reduce a una simple interacción directa con los alumnos (Peña, s. f.).

Un profesor de cátedra no tiene el tiempo para todo esto, tampoco un número reducido de maestros de dedicación completa en una facultad de derecho. Una verdadera facultad de derecho no es solo "tiza, tablero y docente", como se creyó hace años, es mucho más.

- Del porqué los "profesores de tiempo completo" aún no quieren o pueden serlo.

Decimos que es menester que las facultades de derecho cuenten con profesores de tiempo completo, pero estos profesores deben tener unas características especiales. En realidad no cualquier abogado puede enseñar derecho. En primer lugar, un educador debe preocuparse por adquirir herramientas pedagógicas que le permitan hacer docencia fundamentada y dirigida, pues la labor del docente tiene unos fines que este debe tener identificados con precisión. ¿Qué competencias quiere generar en un curso el profesor?, ¿hacia cuál de cada una de ellas se orientan las actividades efectuadas en clase y fuera de clase? A manera de ejemplo, si el profesor no lo tiene claro, se puede dudar de la manera en que enseña el derecho.

El conocimiento del derecho y la enseñanza del mismo son dos cuestiones diferentes, no basta con dominar y ser experto en una materia jurídica para saber "enseñar". Para lograr una adecuada enseñanza, la utilización activa de las herramientas ligadas a las TIC hoy en día se torna indispensable. Además, el uso de la didáctica e instrumentos pedagógicos como el aprendizaje significativo son importantes. Así como también es necesaria la disponibilidad para el diálogo en 
el aula y fuera del aula, ya que no es profesor quien no interactúa con los estudiantes o no admite discusión acerca de sus posturas, pues da el peor de los ejemplos; ni tampoco es educador quien hace de la pedantería su principal estandarte, esos tiempos ya pasaron. El ejercicio "descrestador" de la docencia en que el maestro se paraba frente a sus aprendices a que lo adularan y a hacer gala egoísta de su sapiencia se acabó, hoy en día se encuentran conferencias de los mejores profesores del mundo por Internet. El reto es otro.

Para poder enseñar el derecho no basta con ser abogado, se requieren como mínimo dos cosas adicionales: vocación y formación. Primero vocación, porque la actitud, la disposición hacia la enseñanza es el factor esencial para que el abogado pueda llegar a ser profesor. Quienes ejercen la docencia saben cómo se disfruta de esta (inclusive cuando los necios perturban la clase) y del entorno educativo. Romaña y Begoña (2003) afirman que los docentes universitarios tienen una verdadera identidad profesional, que les hace posible identificarse como grupo, la cual tiene un componente personal y uno social que se entremezclan.

En segundo lugar la formación, por más que le guste a un profesor "dictar clases", para poder "enseñar derecho" y "formar abogados", cuestiones bien distintas, se precisa aprender a enseñar. La práctica es obviamente el primer paso, enfrentarse por primera vez a un grupo de estudiantes es algo que genera pavor. Pero una vez superado, el profesor debe interesarse además por enriquecer sus habilidades en la enseñanza, y esto requiere otros aprendizajes y prácticas que se alejan de lo jurídico. Los hechos demuestran que todavía allí hay mucho por hacer, pues algunos desempeñan la docencia (o dictan clases) con un sentido de la improvisación aterrador, y aún peor, con la complicidad de las facultades donde laboran.

Al respecto Sánchez (s. f.) señala unos obstáculos que tienen los docentes frente a la capacitación: (i) el individualismo y el aislamiento, como característica de ejercicio profesional del docente universitario, dificulta la investigación compartida y las actividades de mejora personal $e$ institucional, que urgen de cooperación y coordinación; (ii) la resistencia al cambio debido, entre otras cuestiones, a: a) la fuerte uniformización de los métodos didácticos; b) la inexistencia de una formación inicial bien fundamentada en la importancia de la innovación educativa; y a c) una enseñanza más centrada en planteamientos teóricos que en procesos de intervención. Ello lleva a que cualquier intento de mejora se enfoque desde una perspectiva de mayor especialización en el campo disciplinar; y iii) la inexistente motivación del profesorado para su optimización docente. A esto el autor le suma un elemento extrínseco:

(...) el "clima laboral", entendido como el conjunto de estímulos que actúan sobre la persona en su lugar de trabajo, y que afectan tanto a las relaciones interpersonales como a la conducta en la organización (González Tirados, 1991). En este sentido, la colaboración entre colegas, en un ambiente de interacción y comunicación constante, es un apoyo moral en el ejercicio docente.Por el contrario, el aislamiento característico del docente hace que sus acciones formativas las realice casi siempre por iniciativa individual, encontrándose luego ciertos obstáculos en su departamento a la hora de llevar a la práctica transformaciones sobre la enseñanza. En este ambiente institucional, no favorable, hay que incluir los problemas que se encuentran en el aula, producto de la apatía y la resistencia al cambio de los alumnos, sobre todo si estas innovaciones le ocasionan mayor trabajo y les afecta a lo que verdaderamente les importa, que son las calificaciones (Sánchez, s. f., p. 1).

Por otro lado, la formación disciplinar, en materia jurídica, también es primordial. En el presente no basta con tener un doctorado, pues dicho título es uno de los mínimos indispensables que deberá tener un profesor de derecho según el MEN. Es claro que el profesor universitario debe supraespecializarse en los temas de su competencia. 
Afortunadamente ya se está superando en Colombia el momento en el cual unos cuantos doctores (profesores con Ph.D.) estaban en las nóminas de dos o más universidades como profesores de tiempo completo, por el solo hecho de tener ese título, práctica que se dio para cumplir los requisitos de acreditación del MEN o tal vez a causa de la mala interpretación que hicieron las facultades del requisito de este ministerio que obliga a las universidades a tener profesores con doctorado, sin verificar que muchos posaron simplemente para la foto del registro calificado o de la acreditación de programas de pregrado o posgrado.

Una vez que una universidad cuenta con "profesores de derecho" con dedicación plena y formación para tal fin, puede comenzar a contar con procesos académicos serios. Pero surgen otras vicisitudes y perversiones de la profesión que les suceden actualmente a los profesores de derecho. En primer lugar, $y$ tal vez la más grave, como no están bien remunerados tienen que dedicarse a otras actividades, como el litigio, la consultoría o la enseñanza en otras instituciones educativas. Esto no sería criticable pues esa praxis jurídica es necesaria, pero ocurre que el desdoblamiento que deben hacer para cumplir con sus múltiples oficios les impide maximizar y mejorar su práctica docente. No debería ser así, pero algunas universidades lo promueven con sus prácticas de contratación laboral nefastas. Las universidades colombianas todavía no entienden la relevancia del asunto, y la calculadora sigue mandando en algunas de ellas. Pasa entonces que el profesor, con maestría y a veces doctorado, tiene que trabajar en dos o más universidades para detentar un salario acorde con su formación.

(ii) La consideración que las universidades tienen de sus docentes.

- Del por qué algunas universidades no comprenden la actividad del profesor de tiempo completo. Si bien dijimos que los profesores deben ser conscientes de perfeccionar su formación jurídica y pedagógica, el principal problema es que las universidades no son conscientes de crear espacios propicios para tener los mejores educadores. Esto parte desde la misma remuneración que le permita al docente dedicarse prevalentemente a su actividad pedagógica. Por lo tanto, los mejores profesores se irán a las universidades que laboralmente les den las condiciones más óptimas, y en sentido opuesto, el resto de los profesores continuará en el ejercicio desenfrenado de la enseñanza. Es una cuestión de selección natural, darwinismo académico.

Pero la cuestión no es solo de remuneración. El docente se motiva en realidad por otros incentivos, sobre todo por aquellos de carácter académico, tales como promoción de viajes de estudios o intercambios académicos, becas para estudios doctorales o posdoctorales, respeto y trato digno a su actividad, fomento a sus publicaciones, etc. Un buen maestro se mueve hoy en redes internacionales de conocimiento y para ello tiene que hablar varios idiomas, viajar, participar en eventos, entre muchas otras actividades. Pero estos estímulos no son regalos o favores que les hacen las universidades a los docentes, se trata de inversión en formación del capital humano que necesariamente dará unos réditos económicos incalculables, pues con docentes más preparados, una facultad de derecho será igualmente prestigiosa y atractiva para un mayor número de estudiantes, si es que desde esa óptica se quiere ver. No en vano en todas las discusiones mediáticas que generan los desastrosos resultados de los estudiantes colombianos en la Pruebas Pisa, se escucha que una de las soluciones para la calidad de la educación radica en la formación de los profesores.

La capacitación interna resulta además vital, es la universidad la que debe facilitar, y es más, obligar a que sus maestros adquieran y refinen su formación pedagógica y disciplinar, de tal modo que dadas las oportunidades, aquel que no se quiera preparar no pueda ascender en escalafón docente. Si la universidad no lo hace, de manera inconsciente tendrá una nómina docente mixta, buenos, regulares y malos profesores, y tal vez estudiantes de las mismas condiciones. Es un asunto que no se le puede dejar al azar. 
Sánchez (s. f.) señala que la formación profesional del profesor universitario tiene varias dificultades, entre ellas:(i) la mercantilización de la universidad, por lo que todos los cambios se dirigen a aspectos de tipo administrativo o técnico, con escasa incidencia en el desarrollo profesional del docente; (ii) cierta insensibilidad administrativa con respecto a la calidad de la enseñanza, ya que esta es poco rentable políticamente al no tener signos tan visibles como otros indicadores de éxito con mayor facilidad cuantificables; (iii) la exigua implicación institucional de la universidad en la formación pedagógica de sus profesionales, al no considerar esta formación como parte de sus prioridades; (iv) el sistema universitario favorece más la investigación que la docencia, de esta forma las actividades pedagógicas tienen poco valor en la selección y promoción del docente y (v)la excesiva carga lectiva que tiene el profesorado, lo cual hace que apenas tenga tiempo para la formación. Aunque este autor también distingue otro conjunto de causas atribuibles al cuerpo docente y que ya indicamos líneas arriba. Por otra parte, es indispensable tener en cuenta que la vocación docente no puede ser fruto únicamente del azar, como todavía acontece, las universidades deben preparar a los relevos generacionales de sus profesores, y deben hacerlo de manera consciente. Se debe escoger entre los mejores estudiantes, y no solo aquellos que sacan las notas más altas, sino entre quienes además desde aprendices del derecho muestran ya vocación y gusto por la enseñanza. Una vez graduados deben contar con el apoyo para prepararse en posgrado en las mejores escuelas y entrar a la práctica docente de modo paulatino. Está probado que el nivel de pertenencia por el alma máter es también clave para el progreso de una escuela de derecho, sin llegar eso sí agrados de endogamia patógena que traen efectos contrarios, todo en su justa medida.

Estas cuestiones que parecen verdades de Perogrullo, por obvias, no lo son en la realidad académica colombiana, y el MEN debería asumir un papel más activo en el asunto, no solamente en materia de acreditación de alta calidad, porque todo lo que se exigepara tal fin debería ser requisito para un simple registro calificado de cualquier programa de derecho. Pero algo está pasando, pues si muchos sabemos que ese es el deber ser y el Ministerio así lo pide en temas de autoevaluación para acreditación de alta calidad, todavía en la mayoría de facultades de derecho no les importa (entre los más de cien programas de derecho con registro calificado en el país solo 27 están acreditados de alta calidad). Algunos juristas ya hablan del descontrol de la educación en Colombia (Reyes, 2014). Nos queda entonces otra duda, además de las cuestionables prácticas de ciertas universidades, ¿̇será que lo que se busca es que en Colombia haya buenas y malas facultades de derecho?

(iii) La situación del profesor investigador.

Si los profesores de derecho de dedicación completa son escasos, más raro aún es encontrar investigadores de derecho, y sobre todo por vocación. Es más, se podría afirmar que muy pocos investigadores en derecho mayores de 35 años en Colombia llegaron a esa labor por vocación propia, la cuestión fue seguramente accidental. Hoy en día, las universidades que asumieron desde un principio con seriedad el trabajo en los centros de investigaciones, en la investigación formativa y en los semilleros de investigación,-procesos a partir de los cuales se generó una escala de jóvenes investigadores-, pueden presentar convincentemente resultados tangibles de esos procesos, y uno de ellos es el de contar con "investigadores" formados desde el pregrado para tal actividad, investigadores por vocación. Este es el deber ser que deben seguir las facultades de derecho.

No obstante, en este aspecto el panorama aún es desolador, muchas universidades contratan como investigadoras a personas sin ningún tipo de formación, y peor aún, no las preparan una vez contratadas. A su vez, algunas entidades educativas pretenden que profesores con vocación de docencia hagan investigación a las malas, asegurando de paso malas prácticas en investigación y resultados deplorables. 
Tampoco es sano que una facultad de derecho tenga únicamente investigadores "paracaidistas", ya que en aquellos lugares donde trataron de formar centros de investigaciones con profesores externos, contratados para investigar por cumplir un formalismo, sin ningún tipo de proceso interno de formación de investigadores propios, el fracaso estuvo a la orden del día. Así mismo, la figura del outsourcing en la investigación que algunas universidades pretenden implantar es desastrosa, servirá tal vez para mostrar resultados pasajeros, pero no para crear procesos y una verdadera cultura de la investigación al interior de una facultad de derecho.

Estas prácticas evidencian que el tema de la investigación se toma todavía a la ligera y además que no hay una debida comprensión de cómo funcionan los procesos de investigación. Reiteramos que la investigación y la formación de investigadores responden a un proceso $y$ toda forma de improvisación o de pragmatismo mercantilista en la búsqueda de resultados es inadecuada. La precipitación y el "resultadismo" no tienen asidero en una academia seria y mucho menos en materia de investigación.

Investigar para un docente trae múltiples ventajas, que tanto profesores como facultades deben entender, en primer lugar, debe valorarse el alto nivel de especialización en una materia que logra un es un ingrediente muy valioso que luego se traduce, no solamente en los escritos del profesor, sino en su práctica docente.

Un docente investigador a diferencia de sus colegas que no lo son, escribe y se supraespecializa en los temas de su investigación, conocimiento que luego traslada al aula de clase. Además, un profesor que escriba contará con una ventaja competitiva importante y muy apreciada en el ámbito de la academia. Adicionalmente, es el ejercicio de la investigación el que genera nuevo conocimiento, el cual constituye la base para la enseñanza del derecho a las nuevas generaciones y ojalá para la solución de problemas que se relacionan con el ámbito jurídico nacional $e$ internacional. Una universidad que no investiga no piensa, y lo mismo puede decirse de una facultad de derecho. Una sociedad que no produce conocimiento está condenada al atraso. Es por eso que el rol del docente investigador necesita ser comprendido y valorado debidamente, no puede seguir viéndose como alguien que le resta dividendos a una universidad, un mal negocio, porque simplemente no factura horas y horas de clase, al tener que pasar tanto tiempo en las bibliotecas o redactando sus escritos.

Un profesor con verdaderas competencias en investigación, pasión por esa labor y resultados concretos en esa actividad, debería ser el recurso humano que cualquier facultad desearía conservar, porque estos personajes no aparecen ni se forman todos los días. Pero tristemente no es así. A la larga es el investigador quien le factura más al prestigio y reconocimiento externo de una universidad, nada que hacer.

Infortunadamente, los sistemas y entidades de validación e indexación de publicaciones nacionales ponen su granito de arena para que menos profesores se dediquen a la investigación, imponiendo estándares importados, a espaldas del contexto colombiano. Es tal el desenfoque que nos comparan con Brasil para justificar sus reformas en la medición de grupos e indexación de revistas, un país que nos lleva muchos años en investigación.

\section{A modo de conclusión}

La calidad académica de los programas de derecho depende en gran medida del papel que ocupe el profesor de derecho en las facultades, de tal suerte que la presencia de profesores de tiempo completo, correctamente capacitados para ejercer esta tarea, es fundamental para crear una verdadera comunidad académica en el seno de la misma. Asumimos que esta debida formación depende de varios factores; primero, se necesita que quienes se dedican a esa actividad la contraigan con franca decisión y se preparen para la enseñanza del derecho en el contexto del siglo XXI. Si anhelamos contar con auténticas facultades de derecho y los profesores queremos 
ocupar el lugar que merecemos socialmente, somos los mismos educadores los que debemos comenzar por hacer valer esa calidad, con un ejercicio docente de alto nivel.

Segundo, es menester que las universidades, incluidas las facultades de derecho, reivindiquen el rol del profesor de derecho, propendiendo porque este adquiera competencias para la debida enseñanza del derecho; así como poniendo a su disposición una serie de incentivos para que su profesión se pueda ejercer con dignidad en el medio jurídico, académico y social. También resulta indispensable que los procesos de formación docente se planeen y ejecuten con miras a satisfacer las necesidades administrativas y académicas de las facultades de derecho.

Las facultades de derecho deben llegar a cumplir una justa combinación entre varias clases de profesores, el de tiempo completo que tiene un rol central en la misma y debe predominar. Dentro de este grupo se debe saber identificar a aquellos que tengan fortalezas y mejores competencias para la investigación, para la enseñanza y para la gestión educativa, para así asignarles tareas de esta naturaleza sin alejarlos en su totalidad de los otros ámbitos de desempeño de la actividad profesoral. A su turno, los profesores de cátedra ocupan un lugar importante gracias a la práctica profesional con que enriquecen a los estudiantes. El rol del docente investigador debe igualmente valorarse por su relevancia en la proyección de una facultad de derecho y en el posicionamiento de la misma en el entorno académico nacional e internacional. Además por ser el eje generador de nuevo conocimiento.

Si todo lo dicho se realiza por parte de los docentes y las universidades, lograremos que el ejercicio profesional de la docencia universitaria en derecho deje de ser una actividad "apéndice", para pasar a ser en realidad valorada y se constituya en el núcleo central de la formación de los abogados del mañana y de la consolidación de genuinas facultades de alta calidad.

Las cosas están cambiando para bien, y esto es un signo positivo, pero todavía se deben superar ciertos obstáculos. Esto sin duda hará que la profesión de profesor de derecho con dedicación completa pueda aportar de manera óptima al ansiado resultado: la mejora de la calidad en la formación jurídica.

\section{REFERENCIAS}

López, L. (2008). El profesor universitario en la contemporaneidad. Revista Facultad de Ciencias Económicas: Investigación y Reflexión, 16(1), 23-40. Recuperado de http://www.scielo.org. co/scielo.php?script $=$ sci_arttext\&pid $=$ S0121$68052008000100003 \& \ln g=e n \&$ thng $=e s$

Molina,C. M. (2014, 5 de agosto).El choque generacional en las facultades de derecho.Recuperado de http://www.ambitojuridico.com/ BancoConocimiento/N/noti-140805-17el_choque_generacional_en_las_facultades_de_derecho/noti-140805-17el_choque_generacional_ en_las_facultades_de_derecho.asp

Peña,J. V. (s. f.).Desarrollo profesional del docente universitario.Recuperado dehttp://www.ub.edu/ histodidactica/index.php?option $=$ com_content\& view $=$ article\&id $=60$ :desarrollo-profesional-deldocente-universitario\&catid $=15 \&$ Itemid $=103$

Reyes, F. (2014, 8 de julio). La educación jurídica también en crisis. Recuperado de http:// www.ambitojuridico.com/BancoConocimiento/N/ noti-140807-08la_educacion_juridica_tambien en_crisis/noti-140807-08la_educacion_juridica_tambien_en_crisis.asp? $\overline{M i g a}=1$

Romaña, T.\&Begoña,G. (2003).La profesión del docente universitario del siglo XXI: ¿̇cambios superficiales o profundos?Revista de Enseñanza Universitaria,21,7-35. Recuperado dehttp://institucional.us.es/revistas/universitaria/21/art\%201. pdf

Sánchez,J. A.(2001). El desarrollo profesional del docente universitario.Revista UDUAL, 22, julio-diciembre. Recuperado de http://www.udual. org/CIDU/Revista/22/DesarrolloProfesional.htm 